\title{
Successful Ultrasound-Guided Gastrografin Enema for Very Low Birth Weight Infants with Meconium-Related Ileus
}

Jaeho Shin, and Ga Won Jeon*

Division of Pediatric Surgery, Department of Surgery, Busan Paik Hospital, Inje University College of Medicine, Busan, Korea Department of Pediatrics*, Busan Paik Hospital, Inje University College of Medicine, Busan, Korea

\section{ABSTRACT}

Purpose: Meconium-related ileus is common in preterm infants. Without proper management, it can cause necrotizing enterocolitis and perforation requiring emergent operation. This study was conducted to describe the efficacy and safety of ultrasoundguided Gastrografin enema at bedside for preterm infants with meconium-related ileus.

Methods: Between March 2013 and December 2014, this study enrolled preterm infants with birth weight $<1,500 \mathrm{~g}$, who were diagnosed with meconium-related ileus requiring ultrasound-guided Gastrografin enema refractory to glycerin or warm saline enemas. Gastrografin was infused until it passed the ileocecal valve with ultrasound guidance at bedside.

Results: A total of 13 preterm infants were enrolled. Gestational age and birth weight were 28.6 weeks (range, 23.9-34.3 weeks) and $893 \mathrm{~g}$ (range, 610-1,440 g), respectively. Gastrografin enema was performed around postnatal day 8 (range, day 3-11). The success rate was $84.6 \%$ ( 11 of 13 cases). Three of these 11 infants received a second procedure, which was successful. Among 2 unsuccessful cases, one failed to pass meconium while the other required surgery due to perforation. The time required to pass meconium was $2.8 \pm 1.5$ hours (range, 1-6 hours). The time until radiographic improvement was 2.8 \pm 3.4 days (range, 1-14 days) after the procedure.

Conclusion: Ultrasound-guided Gastrografin enema at bedside as a first-line treatment to relieve meconium-related ileus was effective and safe for very low birth weight infants. We could avoid unnecessary emergent operation in preterm infants who have high postoperative morbidity and mortality. This could also avoid transporting small preterm infants to fluoroscopy suite.

Key Words: Gastrografin, Intestinal obstruction, Meconium, Preterm infant, Very low birth weight infant
Received: 21 October 2017

Revised: 30 December 2017

Accepted: 21 January 2018

Correspondence to: Ga Won Jeon

Department of Pediatrics, Busan

Paik Hospital, Inje University College of Medicine, 75 Bokji-ro, Busanjin-gu, Busan 47392, Korea

Tel: +82-51-890-6497

Fax: +82-51-895-7785

E-mail: iamgawon@hanmail.net

Copyright(c)

By Korean Society of Neonatology.

All right reserved.

This is an Open-Access article distributed under the terms of the Creative Commons Attribution Non-Commercial License (http://creativecommons.org/licenses/ by-nc/4.0), which permits unrestricted non-commercial use, distribution, and reproduction in any medium, provided the original work is properly cited. 


\section{INTRODUCTION}

Meconium is composed of intestinal epithelial cells, lanugo, mucus, amniotic fluid, bile, and water. It is viscous, thick, and sticky like tar, and becomes inspissated in the intestinal lumen. Therefore, it can obstruct the gastrointestinal tract and cause various diseases ranging from mild and transient functional ileus to severe obstruction requiring surgical intervention ${ }^{1)}$. Meconium-related ileus was first reported by Clatworthy et al. in $1956^{2}$. It is defined as impaired meconium excretion due to impaction, and presents as functional ileus, a distended small intestine (especially distal ileum), and microcolon ${ }^{3)}$. Meconiumrelated ileus is more common in preterm infants than in term infants, especially in extremely preterm infants ${ }^{4,5)}$. Greenholz et al. ${ }^{6)}$ reported that $63 \%$ of infants with meconium-related ileus are preterm.

Impacted meconium can cause progressive abdominal distension, worsening respiratory symptoms due to a distended abdomen, increased intraluminal pressure, bowel ischemia, necrotizing enterocolitis (NEC), and perforation requiring emergent surgery. Considering the high surgical morbidity and mortality rates in preterm infants, unnecessary emergent surgery should be avoided. Noblett first described Gastrografin enema as a therapeutic intervention to avoid surgery in uncomplicated meconium-related ileus ${ }^{7}$. Since then, Gastrografin enema has been used widely for meconium obstruction in neonates, especially in preterm infants.

However, treatment guidelines for preterm infants with meconium obstruction are currently unavailable. Indications for when to start therapeutic Gastrografin enema in preterm infants with meconium obstruction and when to consider surgical intervention instead of Gastrografin enema have not been well established. Moreover, the Gastrografin enema procedure itself can cause perforation of the gastrointestinal tract, epithelial cell damage, dehydration, and electrolyte imbalance due to the high osmolality of Gastrografin ${ }^{8,9)}$. Furthermore, it is difficult to transport small and unstable preterm infants to the fluoroscopy suite.

The objective of this study was to present our experience with ultrasound-guided Gastrografin enema as a therapeutic intervention in very low birth weight preterm infants with meconiumrelated ileus. The procedure is performed at bedside in the incubator in the neonatal intensive care unit without the need for transport to the fluoroscopy suite. We also described the efficacy and safety of Gastrografin enema.

\section{MATERIALS AND METHODS}

\section{Subjects}

The protocol of this study was approved by the Institutional Review Board of Busan Paik Hospital (approval number: 150250). Preterm infants with birth weight $<1,500$ g who were admitted to the neonatal intensive care unit of Busan Paik Hospital between March 2013 and December 2014 were enrolled in the study. The study infants included those diagnosed with meconium-related ileus who were refractory to glycerin or warm saline enemas, and required Gastrografin enemas. Preterm infants with a chromosomal abnormality, congenital gastrointestinal obstruction, or NEC were excluded. These conditions might act as confounding factors by interfering with gastrointestinal function and survival. Therefore, these cases were unsuitable for evaluating the efficacy and safety of Gastrografin enema.

\section{Study protocol}

Clinical data were collected retrospectively from medical records. Meconium-related ileus in preterm infants was diagnosed based on the following criteria: 1) abdominal distension and obstructive symptoms, with difficulty passing additional meconium within a few days after passing initial meconium;2) absence of congenital gastrointestinal obstruction or NEC; and 3) abdominal radiography showing gaseous distension ${ }^{10,11)}$. The diagnosis of meconium-related ileus and the decision to perform Gastrografin enema were left to the attending physician based on patient severity according to clinical symptoms and abdomen radiography.

Gastrografin enema was performed by an experienced pediatric surgeon when meconium obstruction did not resolve after glycerin or warm saline enemas. Gastrografin ${ }^{\circledR}$ (BayerHealthcare, Newbury, England, 2,150 mOsm/L) was mixed with normal saline at a ratio of 1:5 (5 mL of Gastrografin and $25 \mathrm{~mL}$ of normal saline), with a final concentration of $358 \mathrm{mOsm} / \mathrm{L}$. After an 8.0-Fr Foley catheter was positioned at the sigmoid colon and the tip of the catheter was ballooned, $20 \mathrm{~mL} / \mathrm{kg}$ of Gastrografin was infused until it passed the ileocecal valve with ultrasound guidance at the bedside in the neonatal intensive care unit. Immediate portable radiography was used to confirm that Gastrografin had reached the desired level and to detect complications associated with the 
procedure. Follow-up abdominal radiography was performed about 6 hours after Gastrografin enema.

Data on gestational age, birth weight, sex, Apgar score, small for gestational age (SGA), respiratory distress syndrome (RDS), patent ductus arteriosus (PDA), ibuprofen therapy for PDA, concentration of magnesium (Mg), feeding start day, full enteral feeding day (>120 mL/kg/day), initial meconium passage day, and the duration of parenteral nutrition were recorded.

Maternal characteristics including antenatal corticosteroid therapy, antenatal magnesium sulfate $\left(\mathrm{MgSO}_{4}\right)$ therapy, maternal pregnancy-induced hypertension (PIH), gestational diabetes mellitus (GDM), and concentration of Mg were also recorded.

Outcomes included postnatal day of Gastrografin enema administration, hours required to pass meconium after the procedure, days until radiographic improvement of dilated intestine after the procedure, the need for repeat Gastrografin enema, intestinal perforation or other complications associated with the procedure, and mortality associated with the procedure.

\section{RESULTS}

\section{Infant and maternal characteristics}

Infant and maternal characteristics are summarized in Table 1 and Table 2, respectively.

A total of 13 preterm infants with birth weight $<1,500 \mathrm{~g}$ and meconium-related ileus refractory to glycerin or warm saline enemas were treated with Gastrografin enema. Gestational age and birth weight were 28.6 weeks (range, 23.9-34.3 weeks) and $893 \mathrm{~g}$ (range, 610-1,440 g), respectively. Most had PDA (11 of 13, $84.6 \%$ ) and required ibuprofen to close the PDA (9 of 13, 69.2\%). Enteral feeding started on the first postnatal day. Full feeding was achieved at postnatal 28 days. Initial meconium was passed at postnatal 3 days. Parenteral nutrition was infused for 23 days. Most of the mothers were treated with antenatal corticosteroids (12 of $13,92.3 \%$ ) and $\mathrm{MgSO}_{4}$ (11 of 13, 84.6\%). Maternal PIH affected $38.5 \%$ (5 of 13). GDM affected 7.7\% (1 of 13). Concentrations of $\mathrm{Mg}$ in mothers and infants were $5.5 \pm 0.7 \mathrm{mg} / \mathrm{dL}$ and $4.0 \pm 1.5 \mathrm{mg} /$ $\mathrm{dL}$, respectively.

\section{Outcomes associated with Gastrografin enema}

Outcomes associated with Gastrografin enema are summarized in Table 3. Gastrografin enema was performed around day 8 (range, day 3-11), and was successful in 11 (84.6\%) of 13 infants.
Three of these 11 infants successfully underwent a second procedure 4 days after the initial procedure. Of 2 unsuccessful cases, 1 failed to pass meconium while the other required surgery due to procedure-related perforation. Time required to pass meconium was $2.8 \pm 1.5$ hours (range, 1-6 hours) after the procedure. Time until radiographic improvement of dilated intestine was $2.8 \pm 3.4$ days (range, 1-14 days) after the procedure.

Patient 3 could not pass meconium, although several glycerin enemas were performed (Figure 1A). He underwent Gastrografin enema (Figure 1B) on the 5th postnatal day due to severe clinical and radiologic abdominal distension. One hour after the procedure (Figure 1D), he passed meconium. Two days after the procedure, his abdominal distension improved (Figure 1C). Four days after the initial procedure, he underwent a second procedure (Figure 1E) due to recurrent abdominal distension. The distension was relieved 1 day after the second procedure (Figure $1 F)$. He had no difficulty passing meconium after the second

Table 1. Infant Characteristics

\begin{tabular}{lc}
\hline Gestational age (wks) & $28.6 \pm 3.0$ \\
Birth weight (g) & $893 \pm 227$ \\
Gender, male & $9(69.2 \%)$ \\
Apgar score at $1 \mathrm{~min}$ & $5 \pm 2$ \\
Apgar score at $5 \mathrm{~min}$ & $7 \pm 1$ \\
SGA & $5(38.5 \%)$ \\
RDS & $13(100 \%)$ \\
PDA & $11(84.6 \%)$ \\
Ibuprofen therapy & $9(69.2 \%)$ \\
Mg concentration (mg/dL, ref $1.5-2.5 \mathrm{mg} / \mathrm{dL})$ & $4.0 \pm 1.5$ \\
Feeding start day & 1 \\
Full enteral feeding day & $28 \pm 12$ \\
PN duration & $23 \pm 19$ \\
First meconium pass day & $2.6 \pm 1.3$ \\
Gastrografin enema day & $8 \pm 3$
\end{tabular}

The values are presented as number $(\%)$ or mean \pm SD.

Abbreviations: SGA, small for gestational age; RDS, respiratory distress syndrome; PDA, patent ductus arteriosus; PN, parenteral nutrition.

\section{Table 2. Maternal Caracteristics}

\begin{tabular}{lc}
\hline Antenatal corticosteroids & $12(92.3 \%)$ \\
Antenatal magnesium sulfate & $11(84.6 \%)$ \\
Maternal PIH & $5(38.5 \%)$ \\
Maternal GDM & $1(7.7 \%)$ \\
Maternal Mg concentration (mg/dL, ref $1.5-2.5 \mathrm{mg} / \mathrm{dL})$ & $5.5 \pm 0.7$
\end{tabular}

The values are presented as number $(\%)$ or mean \pm SD.

Abbreviations: PIH, pregnancy induced hypertension; GDM, gestational diabetes mellitus. 
Table 3. Outcomes Asociated with Gastrografin Enema

\begin{tabular}{llcccc} 
Pt & Outcome & $\begin{array}{c}\text { Number } \\
\text { of enema }\end{array}$ & $\begin{array}{c}\text { Day of } \\
\text { enema* }\end{array}$ & $\begin{array}{c}\text { Passing } \\
\text { hour }^{\dagger}\end{array}$ & Radiography $^{\ddagger}$ \\
\hline 1 & Successful & 1 & 8 & 3 & 2 \\
2 & Successful & 1 & 5 & 6 & 1 \\
3 & Successful, redo & 2 & $5 / 9$ & $1 / 2$ & $2 / 1$ \\
4 & Unsuccessful & 1 & 10 & Not passed & Not improved \\
5 & Unsuccessful & 1 & 12 & Operation due to perforation \\
6 & Successful & 1 & 9 & 2 & 1 \\
7 & Successful & 1 & 3 & 2 & 2 \\
8 & Successful & 1 & 8 & 3 & 3 \\
9 & Successful, redo & 2 & $11 / 15$ & $5 / 1$ & $1 / 2$ \\
10 & Successful & 1 & 6 & 3 & 2 \\
11 & Successful & 1 & 11 & 1 & 2 \\
12 & Successful, redo & 2 & $5 / 7$ & $3 / 4$ & Not improved/7 \\
13 & Successful & 1 & 10 & 2 & 3 \\
\hline
\end{tabular}

* Postnatal days when Gastrografin enema was done (first/second). ${ }^{\dagger}$ Hours required to pass meconium after Gasrografin enema (first/ second).

${ }^{\ddagger}$ Days required to improve at radiography after Gastrografin enema (first/second). procedure.

Patient 4 could not pass meconium after the first procedure. However, she could not undergo a second procedure due to poor general condition associated with systemic infection. She died a few days later due to sepsis.

Patient 5 was born at 26.3 weeks of gestation with birth weight of 1,010 g. His Apgar scores at 1 and 5 minutes were 3 and 7, respectively. There was no history of maternal PIH, GDM, or antenatal $\mathrm{MgSO}_{4}$ therapy. Abdominal distension appeared on postnatal day 3 and progressed. He had problem to pass meconium after passing very small amount of meconium. He was treated with ibuprofen on postnatal days 9-11 to close a hemodynamically significant PDA. He underwent Gastrografin enema at postnatal 12 days due to severe abdominal distension with body weight of $720 \mathrm{~g}$ (23\% weight loss compared to birth weight). Immediate portable radiography detected a pneumoperitoneum. He underwent laparotomy. We found a small perforation in the distal jejunum without evidence of NEC, jejunal atresia, or ileal atresia.
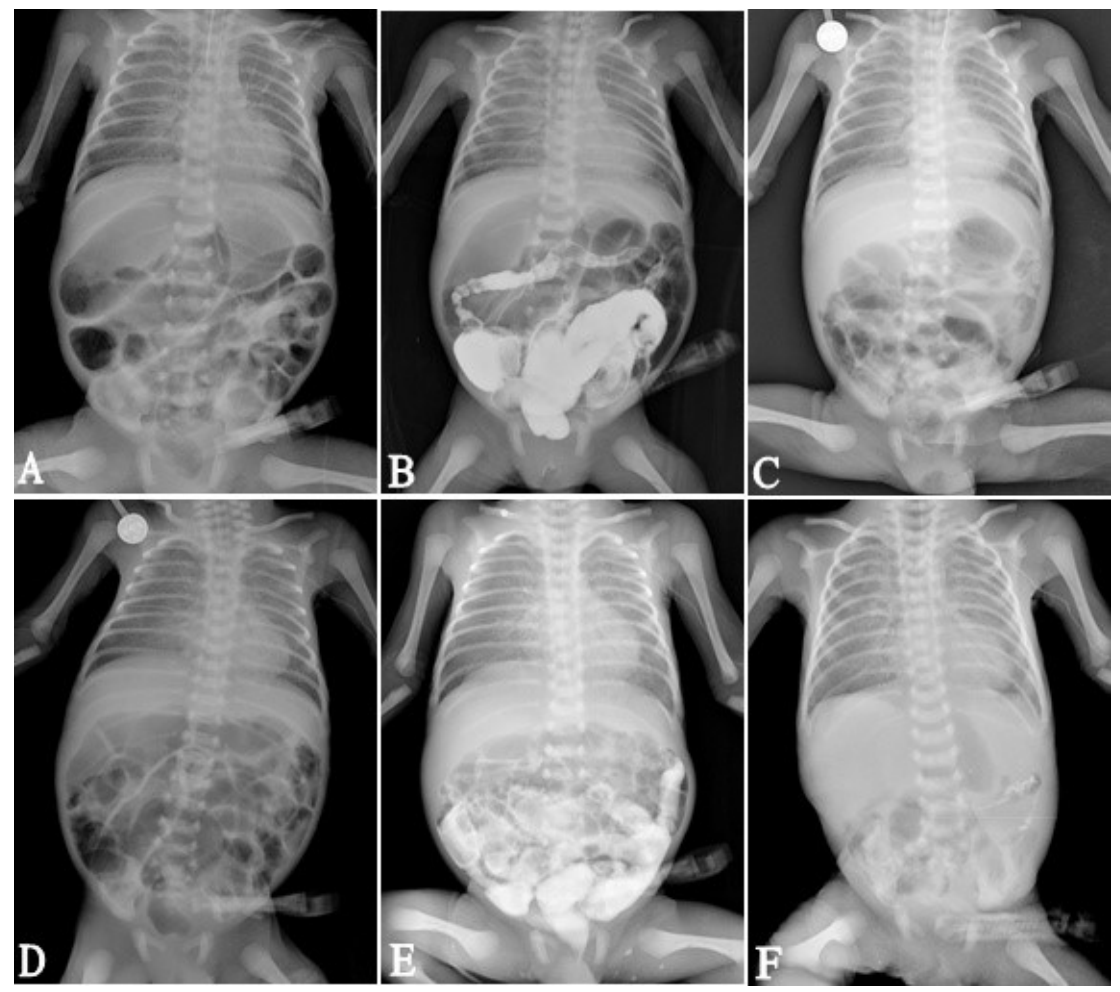

Figure 1. Radiograph of patient 3. Radiograph of patient 3 shows dilated ileal loop at postnatal 4 days before Gastrografin enema (A), dilated ileal loop during Gastrografin enema at postnatal 5 days (B), disappearance of dilated ileal loop after procedure at postnatal 7 days $(\mathrm{C})$, recurrent abdominal distension and dilated ileal loop 4 days after the initial procedure (D), second Gastrografin enema at postnatal 9 days (E), and disappearance of dilated ileal loop (F). 
Gastrografin enema was performed at postnatal 5 days in patient 12 due to severe clinical and radiologic abdominal distension. Three hours after the procedure, he passed meconium. Abdominal distension was improved clinically but not radiologically. Distension recurred 2 days after the procedure, and Gastrografin enema was repeated. Radiologic abdominal distension was improved at 7 days after the second procedure.

Patient 13 could not pass additional meconium after passing a small amount (Figure 1A). He underwent Gastrografin enema on postnatal day 10 (Figure 2B) due to severe clinical and radiologic abdominal distension. Two hours after the procedure, he passed a large amount of plugged meconium. Three days after the procedure, abdominal distension was markedly improved (Figure 2C).

\section{DISCUSSION}

Meconium-related ileus in preterm infants is associated with intestinal immaturity and bowel dysmotility, and is not simply an obstruction caused by sticky meconium ${ }^{12)}$. The migrating motor complex (or migrating myoelectric complex, MMC) is a caudally-propagated sequence of smooth muscle contraction in the intestine caused by high-amplitude impulses. Frequency, amplitude, and duration of MMC mature after 34-35 weeks of gestation. Therefore, preterm infants delivered before maturation of the MMC are at high risk of meconium-related ileus ${ }^{13)}$.

The interstitial cells of Cajal (ICC) play a pacemaker role in
MMC. Immature ICC will result in immature MMC, which is associated with meconium obstruction ${ }^{14)}$. Toyosaka et al. ${ }^{15)}$ reported that intramural ganglion cells are immature at ileostomy. These immature ganglion cells appear mature a few weeks or months later at ileostomy repair. The authors suggested that immature intramural ganglion cells are the main cause of meconium ileus ${ }^{15)}$.

Ziegler and Del Pin suggested that meconium-related ileus in cystic fibrosis patients is associated with maternal DM, $\mathrm{PIH}$, and chronic hypertension diagnosed before pregnancy ${ }^{16,17)}$. Prenatal risk factors such as maternal DM, PIH, intrauterine growth retardation, and perinatal asphyxia can cause intestinal hypoperfusion and ischemia associated with intestinal dysmotility that causes meconium ileus ${ }^{1,18)}$. Maternal PIH and SGA were each found in $38.5 \%$ of cases in our study.

Inspissated intraluminal thick meconium and intestinal dysmotility cause meconium-related ileus. Postnatal risk factors such as RDS, PDA, intraventricular hemorrhage, asphyxia, and sepsis are also associated with intestinal hypoperfusion or ischemia, resulting in intestinal dysmotility and meconium-related ileus ${ }^{19,20)}$. All patients had RDS and most (84.6\%) had PDA in our study. The concentration of Mg was $4.0 \mathrm{mg} / \mathrm{dL}$. Hypermagnesemia is known to suppress MMC in the intestine and cause meconiumrelated ileus ${ }^{1,18)}$. Fetal hyperglycemia resulting from maternal DM can increase glucagon production, suppress MMC and intestinal smooth muscle contraction, and cause meconium-related ileus in the same manner.

Gastrografin is a combination of diatrizoate meglumine and
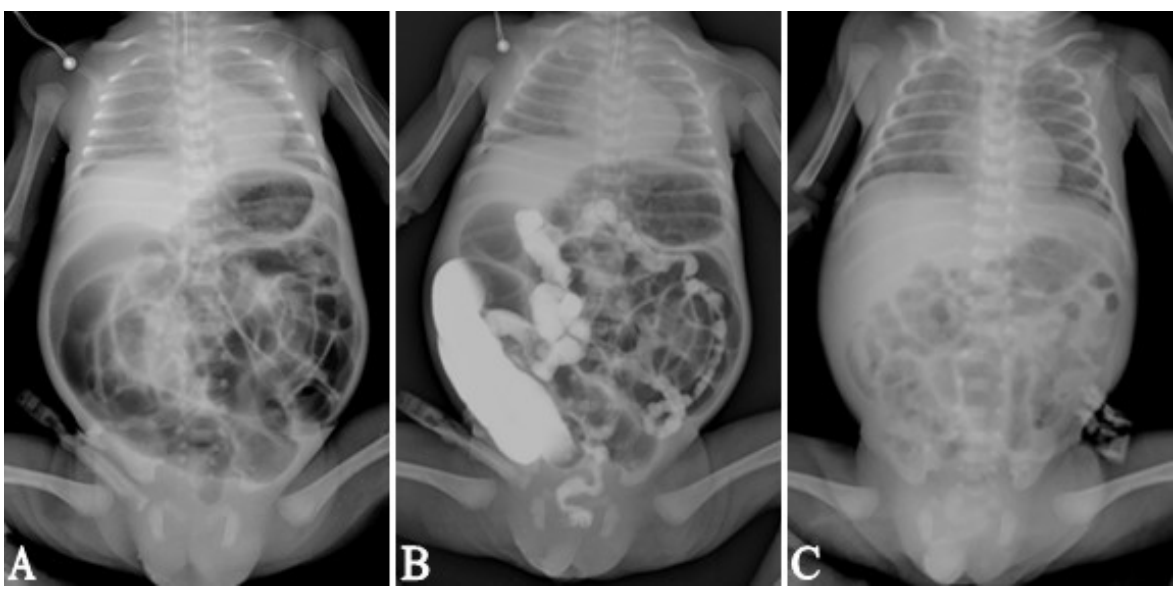

Figure 2. Radiograph of patient 13. Radiograph of patient 13 shows dilated ileal loop at postnatal 10 days before Gastrografin enema (A), dilated ileal loop during Gastrografin enema on the same day (B), and disappearance of dilated ileal loop 3 days after procedure at postnatal 13 days $(\mathrm{C})$. 
diatrizoate sodium salts. It is a high-osmolality radiocontrast agent containing iodine. Gastrografin enema as a therapeutic intervention for meconium obstruction has been used for neonates, especially in preterm infants, when there is failure to pass meconium ${ }^{4,21)}$. Complications of Gastrografin enema in preterm infants such as intestinal perforation, hypotension, dehydration, electrolyte imbalance, epithelial cell damage, and NEC due to high osmolality have been reported ${ }^{8,9,22,23)}$. One (7.7\%) patient had intestinal perforation after the procedure in our study. He underwent Gastrografin enema 1 day after ibuprofen treatment for PDA. We detected perforation in the distal jejunum. Although intestinal perforation was noticed immediately after the procedure, he had risk factors for spontaneous bowel perforation such as PDA, ibuprofen treatment, and low Apgar scores. The catheter was positioned at the sigmoid colon and Gastrografin was infused until it passed the ileocecal valve, which was not adjacent to the perforation site. Therefore, we could not determine an association between the intestinal perforation and the procedure.

Success of Gastrografin enema in preterm infants was associated with gestational age, birth weight, and body weight at the time of the procedure, reflecting maturation of intestinal motility and $\mathrm{MMC}^{24)}$. In 1 unsuccessful case in our study, body weight at the time of the procedure was $720 \mathrm{~g}$, in contrast with a birth weight of $1,010 \mathrm{~g}$ at a gestational age of 26.3 weeks. The loss in birth weight was $23 \%$.

Hatanaka et al. recently reported that obstruction was resolved with Gastrografin enema at 14 postnatal days in 12 of 14 extremely low birth weight infants with meconium-related ileus. Two successful cases underwent surgery. These were diagnosed with congenital megacolon and congenital ileal atresia. Therefore, the success rate of Gastrografin enema was $100 \%$, except in those with congenital intestinal obstruction. Therefore, Gastrografin enema can be safely performed without intestinal perforation, especially in extremely preterm infants ${ }^{25)}$.

The success rate of Gastrografin enema in very low birth weight infants with meconium obstruction was reportedly $70 \%$ in 8 of 13 cases $^{18)}, 80 \%$ in 8 of 10 cases $^{4)}$, and $>80 \%{ }^{26)}$ in cases of small intestinal obstruction. The success rate was $100 \%$ (all 20 patients) in cases of colonic obstruction ${ }^{11)}$. In the present study, the success rate of Gastrografin enema in very low birth weight infants with meconium-related ileus was $84.6 \%$.

Gastrografin enema was effective, safe, and repeatable. There were no procedure-related complications in stable very low birth weight infants. However, the procedure was ineffective in unstable very low birth weight infants ${ }^{27)}$ and may cause deterioration of patient status or require more time to evacuate meconium.

In a study of 1,236 infants with meconium ileus in 39 pediatric radiology departments, intestinal perforation after Gastrografin enema was associated with the ballooning of the catheter tip, rather than with the procedure itself or the osmolality of Gastrografin $^{28)}$. Success rates were higher with Gastrografin, regardless of the osmolality of the radiocontrast agent or size of the cathe$\operatorname{ter}^{28)}$.

All 13 patients in our study were intubated and needed ventilator care, and all had central venous catheters for parenteral nutrition. They were at risk for extubation, air leaks, pneumothorax, hypoxemia, hypercapnia, or disconnection of the central venous catheter with transfer to a transport incubator and then to the fluoroscopy suite. They also were at risk for hypothermia, metabolic acidosis, and other complications related to transfer outside of an incubator that maintained proper temperature and humidification. Our results suggest that performing ultrasoundguided Gastrografin enema at the bedside in the incubator without transport to the fluoroscopy suite is convenient. This method does not adversely affect the preterm infant, thus decreasing complications related to transfer.

Patient 3 underwent Gastrografin enema on postnatal day 5 and needed parenteral nutrition for 11 days, reaching full feeding at postnatal day 15. On the other hand, patient 13 underwent Gastrografin enema on postnatal day 10 and needed parenteral nutrition for over 1 month, reaching full feeding at postnatal 1 month. Delayed treatment of meconium-related ileus can cause complications such as intestinal perforation, NEC, or sepsis ${ }^{10,24)}$. Therefore, evacuating impacted meconium with Gastrografin enema in the early postnatal period can improve meconiumrelated ileus, and prevent bowel ischemia, NEC, and bowel perforation. The time to full feeding is shortened, decreasing the duration of parenteral nutrition and central venous catheter use, thereby decreasing the risk of parenteral nutrition-associated cholestasis and central catheter-associated sepsis.

Although treatment guidelines for preterm infants with meconium-related ileus do not exist, prompt diagnosis is very important for successful management to achieve better outcomes. Early postnatal diagnosis requires a high index of suspicion based on risk factors, clinical symptoms, and radiologic findings. Ultrasound-guided Gastrografin enema performed by an expe- 
rienced pediatric surgeon at the bedside was effective and safe in very low birth weight infants as a first-line treatment to relieve meconium-related ileus. The procedure can avoid unnecessary emergent surgery in preterm infants with high risk of postoperative morbidity and mortality.

\section{REFERENCES}

1) Emil S, Nguyen T, Sills J, Padilla G. Meconium obstruction in extremely low-birth-weight neonates: guidelines for diagnosis and management. J Pediatr Surg 2004;39:731-7.

2) Clatworthy HW, Jr., Howard WH, Lloyd J. The meconium plug syndrome. Surgery 1956;39:131-42.

3) Kubota A, Shiraishi J, Kawahara H, Okuyama H, Yoneda A, Nakai H, et al. Meconium-related ileus in extremely low-birthweight neonates: etiological considerations from histology and radiology. Pediatr Int 2011;53:887-91.

4) Shinohara T, Tsuda M, Koyama N. Management of meconiumrelated ileus in very low-birthweight infants. Pediatr Int 2007;49: 641-4.

5) Kubota A, Imura K, Yagi M, Kawahara H, Mushiake S, Nakayama $\mathrm{M}$, et al. Functional ileus in neonates: Hirschsprung's diseaseallied disorders versus meconium-related ileus. Eur J Pediatr Surg 1999;9:392-5.

6) Greenholz SK, Perez C, Wesley JR, Marr CC. Meconium obstruction in markedly premature infant. J Pediatr Surg 1996;31: 117-20.

7) Noblett HR. Treatment of uncomplicated meconium ileus by Gastrografin enema: a preliminary report. J Pediatr Surg 1969; 4:190-7.

8) Ein SH, Shandling B, Reilly BJ, Stephens CA. Bowel perforation with nonoperative treatment of meconium ileus. J Pediatr Surg 1987;22:146-7.

9) Chang PY, Huang FY, Yeh ML, Sheu JC, Chen BF, Chen CC. Meconium ileus-like condition in Chinese neonates. J Pediatr Surg 1992;27:1217-9.

10) Dimmitt RA, Moss RL. Meconium diseases in infants with very low birth weight. Semin Pediatr Surg 2000;9:79-83.

11) Vinograd I, Mogle P, Peleg O, Alpan G, Lernau OZ. Meconium disease in premature infants with very low birth weight. J Pediatr 1983;103:963-6.

12) Milla PJ. Development of intestinal structure and function in neonatal gastroenterology. In: Tanner MS, Stocks RJ, editors. Neonatal gastroenterology: contemporary issues. Newcastle upon Tyne(UK): Intercept, 1984;1-20.

13) Wolinski J, Slupecka-Ziemilska M, Boryczka M, Grzesiak $P$, Kwiatkowski J, Kotarba G. Small intestine motility development in newborn mammals. Dev Period Med 2016;20:53-60.

14) Yoo SY, Jung SH, Eom M, Kim IH, Han A. Delayed maturation of interstitial cells of Cajal in meconium obstruction. J Pediatr Surg 2002;37:1758-61.

15) Toyosaka A, Tomimoto Y, Nose K, Seki Y, Okamoto E. Immaturity of the myenteric plexus is the aetiology of meconium ileus without mucoviscidosis: a histopathologic study. Clin Auton Res 1994;4:175-84.

16) Ziegler MM. Meconium ileus. Curr Probl Surg 1994;31:731-77.

17) Del Pin CA, Czyrko C, Ziegler MM, Scanlin TF, Bishop HC. Management and survival of meconium ileus. A 30-year review. Ann Surg 1992;215:179-85.

18) Garza-Cox S, Keeney SE, Angel CA, Thompson LL, Swischuk LE. Meconium obstruction in the very low birth weight premature infant. Pediatrics 2004;114:285-90.

19) Blott M, Greenough A, Gamsu HR, Nicolaides K, Campbell $\mathrm{S}$. Antenatal factors associated with obstruction of the gastrointestinal tract by meconium. Br Med J (Clin Res Ed) 1988;296: 250.

20) Yu HY, Shin JE, Eun HS, Lee SM, Park MS, Lee MJ, et al. Meconium-related ileus in extremely low-birth weight newborn infants. Neonatal Med 2013;20:121-8.

21) Krasna IH, Rosenfeld D, Salerno P. Is it necrotizing enterocolitis, microcolon of prematurity, or delayed meconium plug? A dilemma in the tiny premature infant. J Pediatr Surg 1996;31: 855-8.

22) Maneksha FR, Betta J, Zawin M, Poppers PJ. Intraoperative hypoxia and hypotension caused by gastrografin-induced hypovolemia. Anesthesiology 1984;61:454-6.

23) Rowe MI, Furst AJ, Altman DH, Poole CA. The neonatal response to gastrografin enema. Pediatrics 1971;48:29-35.

24) Cho HH, Cheon JE, Choi YH, Lee SM, Kim WS, Kim IO, et al. Ultrasound-guided contrast enema for meconium obstruction in very low birth weight infants: fctors that affect treatment success. Eur J Radiol 2015;84:2024-31.

25) Hatanaka A, Nakahara S, Takeyama E, Iwanaka T, Ishida K. Management of extremely low birth weight neonates with bowel obstruction within 2 weeks after birth. Surg Today 2014;44:226974.

26) Fuchs JR, Langer JC. Long-term outcome after neonatal meconium obstruction. Pediatrics 1998;101:E7.

27) Koshinaga T, Inoue M, Ohashi K, Sugito K, Ikeda T, Tomita R. Therapeutic strategies of meconium obstruction of the small bowel in very-low-birthweight neonates. Pediatr Int 2011;53: 338-44.

28) Kao SC, Franken EA, Jr. Nonoperative treatment of simple meconium ileus: a survey of the Society for Pediatric Radiology. Pediatr Radiol 1995;25:97-100. 